\title{
Investigation of non-linear acoustic losses at the open end of a tube
}

\author{
James M. Buick ${ }^{\text {a) }}$ \\ Department of Mechanical and Design Engineering, University of Portsmouth, Anglesea Road, \\ Portsmouth P01 3DJ, United Kingdom \\ M. Atig \\ Laboratoire d'Acoustique de l'Université du Maine, UMR CNRS 6613, Avenue Olivier Messiaen, \\ 72085 le Mans Cedex 9, France \\ D. J. Skulina and D. M. Campbell \\ School of Physics, The University of Edinburgh, Kings Buildings, Mayfield Road, Edinburgh \\ EH9 3JZ, United Kingdom \\ J. P. Dalmont and J. Gilbert \\ Laboratoire d'Acoustique de l'Université du Maine, UMR CNRS 6613, Avenue Olivier Messiaen, \\ 72085 le Mans Cedex 9, France
}

(Received 1 March 2010; revised 9 September 2010; accepted 27 December 2010)

\begin{abstract}
At high acoustic level, non-linear losses at the end of a tube are usually interpreted as the consequence of a jet formation at the tube end resulting in annular vortices dissipating part of the acoustic energy. Previous work has shown that two different regimes may occur. The present work, using particle image velocimetry visualization, lattice Boltzmann method simulation in $2 \mathrm{D}$, and an analytical model, shows that the two different regimes correspond to situations for which the annular vortices remain attached to the tube (low acoustic particle velocity) or detached (high acoustic particle velocity). (C) 2011 Acoustical Society of America. [DOI: 10.1121/1.3543987]
\end{abstract}

PACS number(s): 43.28.Py [AH]

Pages: $1261-1272$

\section{INTRODUCTION}

It is known that geometrical singularities in tubes, such as orifices or the open end, may induce non-linear losses. ${ }^{1-9}$ Studying the specific cases of a straight tube, Disselhorst and Van Wijngaarden ${ }^{10}$ interpreted that these losses as due to the formation of a jet at the end of the tube resulting in annular vortices. Peters et al. ${ }^{11}$ showed that the level of non-linear losses depends on the shape of the end of the tube. This was confirmed by the experimental work of Atig et al., ${ }^{12}$ which also suggested that a change of regime may occur at a particular acoustic particle velocity. This change of regime was evident from a discontinuity which was observed in the termination impedance curve. The acoustic particle velocity at which the change of regime occurred was also observed to depend on the shape of the open end of the tube.

The aim of the present work is to corroborate previous observation and to identify the nature of the change of regime, and understand how it may affect non-linear losses. Experiments have been performed using particle image velocimetry (PIV) in order to visualize the vortices at the end of the tube. Additionally, two-dimensional (2-D) lattice Boltzmann method (LBM) simulations were run to investigate whether such simulations are able to reproduce qualitatively the observed phenomena. Finally, a simplified analytical model, based on observations from the PIV experiments and incorporating vortex-sound theory, ${ }^{13}$ was

\footnotetext{
a) Author to whom correspondence should be addressed. Electronic mail: james.buick@port.ac.uk
}

developed in order to understand how the change of regime may induce a change in the relationship between non-linear losses and acoustic particle velocity.

\section{BACKGROUND THEORY}

In linear acoustics, at low frequencies such that the Helmholtz number $H e=k a \ll 1$, where $k$ is the wavenumber of the acoustic signal and $a$ is the radius of the tube; the radiation impedance or termination impedance, $Z_{r}$, is given for an unflanged ${ }^{14}$ and for a infinitely flanged ${ }^{15-17}$ tubes by

$$
\frac{Z_{r}}{Z_{c}}= \begin{cases}\frac{1}{4}(k a)^{2}+j k \delta_{0} & \text { unflanged } \\ \frac{1}{2}(k a)^{2}+j k \delta_{\infty} & \text { infinitely flanged }\end{cases}
$$

Here $Z_{c}=\rho_{0} c_{0} / S$ is the characteristic impedance of air with the ambient density $\rho_{0}$ and speed of sound $c_{0}$, and $S$ is the cross-sectional area of the tube; $\delta_{0}=0.6133 a$ (Ref. 14) and $\delta_{\infty}=0.8216 a$ (Ref. 15). Expressions for pipes of finite thickness are given by Dalmont et al. ${ }^{16}$ The imaginary part of the termination impedance relates to the fluid inertia around the end of the tube while the real part is the radiation resistance corresponding to the energy dissipated after being radiated from the open end. In the non-linear case the termination impedance can be calculated in the same manner using the fundamental harmonic, since the level of harmonic distortion is generally small at the open end of a tube.

Modeling of the non-linear case suggests that the termination impedance can be modified by the addition of an extra term due to the non-linear effects, $Z_{n l}: Z_{t}=Z_{r}+Z_{n l}$. At high 
Strouhal numbers $\left(S t=\omega a / u_{\mathrm{ac}}\right.$, where $\omega$ is the angular frequency of the sound and $u_{\mathrm{ac}}$ is the acoustic particle velocity) this is given by ${ }^{10}$

$$
\frac{Z_{n l}}{Z_{c}}=\beta M(\mathrm{St})^{1 / 3},
$$

where $M=u_{\mathrm{ac}} / c_{0}$ is the acoustic Mach number. Disselhorst and Van Wijngaarden ${ }^{10}$ performed numerical simulations and found a value of $\beta$ between 0.6 and 1.0, while Peters and Hirschberg $^{18}$ obtained a value of 0.2 . For low Strouhal numbers Peters et al. ${ }^{11}$ gave the correction term as

$$
\frac{Z_{n l}}{Z_{c}}=\frac{2 c_{d}}{3 \pi} M,
$$

where $C_{d}$ is 2 for a thin-walled unflanged tube and 13/9 for a flanged tube.

Atig et al. ${ }^{12}$ measured the termination impedance using the two-microphone method. A number of tubes were studies which were identical except for the inside edge of the open end, which had a prescribed radius of curvature, $r$ (see Fig. 2) ranging from $r<0.01 \mathrm{~mm}$ (approximately a right angle) to $r=4 \mathrm{~mm}$. The real part of the termination impedance, representing the dissipation of energy from the open end of the tube, was presented and is reproduced in Fig. 1. For $r<0.001 \mathrm{~mm}$ the measurements agree well with Eq. (3) (with $c_{d}=2$ ) for acoustic particle velocities $<10.6 \mathrm{~ms}^{-1}$. At this acoustic particle velocity a change of regime was observed such that there was a discontinuity in $\Re\left(Z_{t} / Z_{c}\right)$. At higher acoustic particle velocities the measurements did not agree with Eq. (3). ${ }^{12}$ Similar changes of regime were also observed for $r=0.3 \mathrm{~mm}$ and $r=1 \mathrm{~mm}$, where a smaller discontinuity was seen. The acoustic particle velocity at which the change of regime occurred was seen to increase with the radius of curvature. ${ }^{12}$

\section{VORTEX-SOUND THEORY}

Vortex-sound theory was developed by Powell ${ }^{13}$ for unconfined subsonic flows. The velocity, $\boldsymbol{v}$ of an inviscid fluid of density $\rho$ under the action of a pressure $p$ is governed by the continuity equation

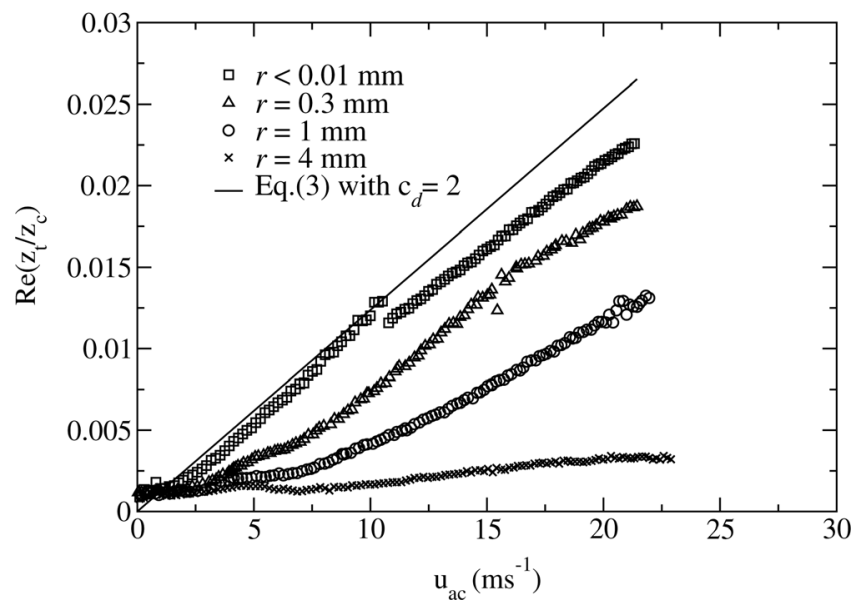

FIG. 1. The real part of the termination impedance for four different terminations, (Adapted from Ref. 12).

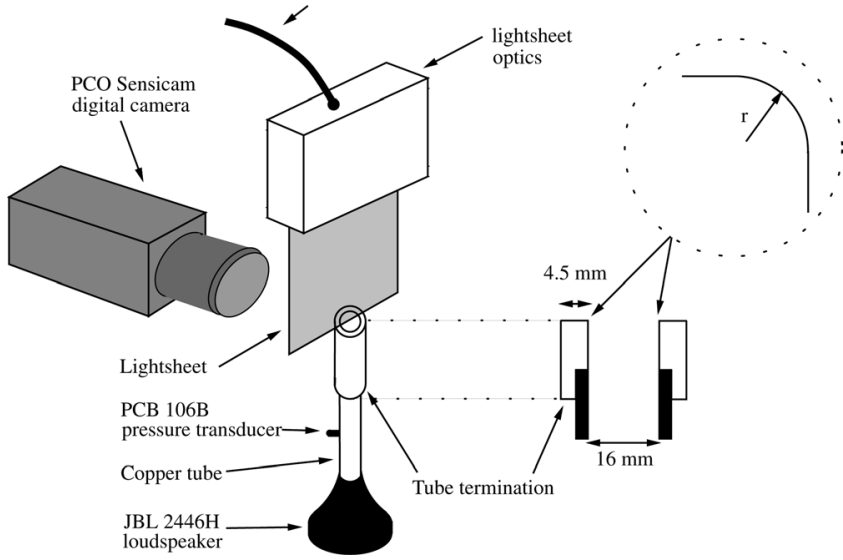

FIG. 2. The experimental configuration.

$$
\frac{\partial \rho}{\partial t}+\nabla \cdot(\rho \boldsymbol{v})=0
$$

and the Euler equation which can be expressed in terms of the vorticity, $\Omega=\nabla \times v$, as,

$$
\rho \frac{\partial \boldsymbol{v}}{\partial t}+\frac{1}{2} \rho \nabla \boldsymbol{v}^{2}+\rho(\Omega \times \boldsymbol{v})+\nabla p=0 .
$$

The inviscid assumption is valid at high Reynolds numbers $R e=a v / v / \gg 1$, where $v$ is the kinematic viscosity. Combining the temporal derivative of Eq. (4) and the divergence of Eq. (5) gives the propagation equation

$$
\begin{aligned}
\nabla^{2} p-\frac{1}{c_{0}^{2}} \frac{\partial^{2} p}{\partial t^{2}}= & -\nabla \cdot\left[\rho(\Omega \times \boldsymbol{v})+\nabla\left(\frac{1}{2} \rho|\boldsymbol{v}|^{2}\right)\right. \\
& \left.-\boldsymbol{v} \frac{\partial \rho}{\partial t}-\frac{1}{2}|\boldsymbol{v}|^{2} \nabla \rho\right]
\end{aligned}
$$

where the homentropic equation of state

$$
\frac{D \rho}{D t}=\frac{1}{c_{0}^{2}} \frac{D p}{D t}
$$

has been used. Equation (6) can be simplified by considering the order of magnitude of each source term on the right hand side. The terms on the right hand side of Eq. (6) scale as ${ }^{13,19}$

$$
\begin{aligned}
\rho(\Omega \times \boldsymbol{v}): \nabla\left(\frac{1}{2} \rho|\boldsymbol{v}|^{2}\right) & : \boldsymbol{v} \frac{\partial \rho}{\partial t}: \frac{1}{2}|\boldsymbol{v}|^{2} \nabla \rho \\
& \sim 1: 1: S t M^{2}: M^{2} .
\end{aligned}
$$

Further, for a compact source $(H e \ll 1)$ at low Mach number $\left(M=u_{\mathrm{ac}} / c_{0} \ll 1\right)$ the contribution of the second source term in Eq. (6) is negligible with respect to the first due to conservation of kinetic energy in an inviscid fluid. ${ }^{19-21}$ Thus in the limit $M \ll 1, S t \ll 1, H e \ll 1, R e \gg 1$, the propagation equation can be expressed as

$$
\nabla^{2} p-\frac{1}{c_{0}^{2}} \frac{\partial^{2} p}{\partial t^{2}}=-\nabla \cdot\left[\rho_{0}(\Omega \times v)\right]
$$

where in the same limit $\rho \simeq \rho_{0}$. 
Conservation of instantaneous acoustic energy is described by Kirchhoff's equation ${ }^{22}$

$$
\frac{\partial E}{\partial t}+\nabla \cdot \mathbf{I}=\mathbf{F} \cdot \mathbf{u}_{\mathrm{ac}},
$$

where

$$
E=\frac{1}{2} \rho_{0}\left|\mathbf{u}_{\mathrm{ac}}\right|^{2}+\frac{1}{2} \frac{p^{2}}{\rho_{0} c_{0}^{2}} \text { and } \mathbf{I}=p \mathbf{u}_{\mathrm{ac}}
$$

are the instantaneous total energy density and acoustic intensity, respectively. $\mathbf{F}$ is an external. Here the instantaneous acoustic particle velocity is defined from the non-stationary part of the velocity potential,

$$
\mathbf{u}_{\mathrm{ac}}=\nabla \phi_{\mathrm{ac}}(t),
$$

where

$$
\boldsymbol{v}=\nabla\left(\phi_{0}+\phi_{\mathrm{ac}}(t)\right)+\nabla \times \boldsymbol{\psi},
$$

$\phi_{0}$ is the time independent part of the velocity potential, and $\psi$ is the streamfunction. For a periodic acoustic field the time-averaged power generated (absorbed) inside a volume $V$ by an external force $\mathbf{F}$ is

$$
\langle\mathcal{P}\rangle=\oint_{S}\langle\mathbf{I} \cdot \mathbf{n}\rangle d_{s}=\oint_{V}\left\langle\mathbf{F} \cdot \mathrm{u}_{\mathrm{ac}}\right\rangle \mathrm{d} V,
$$

where $\langle X\rangle=\frac{1}{T} \int_{0}^{T} X \mathrm{~d} t$ is the time-average value of $X$ over a period and $\mathbf{n}$ is an outward normal. Following Howe, ${ }^{23}$ the external force $\mathbf{F}$ can be identified with the Coriolis force term in Eq. (9), $\mathbf{F}_{c}=-\rho_{0}(\Omega \times \mathbf{v})$ to give

$$
\mathcal{P}_{s}=-\rho_{0} \oint_{V}(\Omega \times \boldsymbol{v}) \cdot \mathbf{u}_{\mathrm{ac}} \mathrm{d} V .
$$

At the open end of a tube of surface area $S$ the time-averaged power is given by

$$
\langle\mathcal{P}\rangle=\langle\operatorname{Sp}(t) u(t)\rangle,
$$

where the acoustic pressure and velocity are expressed using the linear wave assumption as

$$
p(t)=p_{\mathrm{ac}} \sin (\omega t) \text { and } u(t)=u_{\mathrm{ac}} \sin (\omega t+\xi) .
$$

Thus

$$
\langle\mathcal{P}\rangle=\Re\left(Z_{t}\right) \frac{S u_{\mathrm{ac}}^{2}}{2},
$$

where $Z_{t}=p_{\mathrm{ac}} e^{-j \xi} / u_{\mathrm{ac}}$ at the surface $S$ and $\Re\left(Z_{t}\right)$ is the real part of $Z_{t}$. When the amplitude of the acoustic waves is large, non-linear effects will appear. In this case Eq. (18) can be applied where it is understood that $u_{\mathrm{ac}}$ and $p_{\mathrm{ac}}$ are, respectively, the acoustical velocity and pressure of the fundamental. Combining Eqs. (15) and (18) and taking $\langle\mathcal{P}\rangle=-\left\langle\mathcal{P}_{s}\right\rangle$ to account for energy absorption gives

$$
\Re\left(\frac{Z_{t}}{Z_{c}}\right)=-\frac{2\left\langle\mathcal{P}_{s}\right\rangle}{S Z_{c} u_{\mathrm{ac}}^{2}}
$$

\section{PIV MEASUREMENT OF THE ACOUSTIC, VELOCITY, AND VORTICITY FIELDS AT THE OPEN END OF A RESONATING TUBE}

In this section the measurement of the velocity (and hence vorticity) and the acoustic fields at the open end of a resonating tube using particle image velocimetry (PIV) is considered. The real part of the termination impedance is then calculated from these fields using vortex-sound theory.

PIV is a non-intrusive technique which measures fluid velocity simultaneously at a number of points in a planar region of space. The technique has been applied to a range of applications including the measurement of both steady and oscillatory flows in air. ${ }^{24-29}$ The basic principle of PIV for measuring the $2 \mathrm{D}$ velocity of a fluid in a plane of interest is relatively simple. The fluid is "seeded" with tracer particles which are assumed to follow the flow. The particles within the plane containing the region of interest are then illuminated twice by a thin pulsed lightsheet. The light is scattered by the particles and captured by a digital camera which records the position of each particle at the time of each light pulse. By measuring the displacement of the particles over the time between the illuminations the velocity can be found. This final step is typically performed using a cross-correlation statistical method rather than tracing each particle individually. The following subsection describes the PIV system applied here. A wider overview can be found in Refs. 30-33.

\section{A. Experimental setup}

The main features of the system are shown in Fig. 2. The acoustic field was generated by a JBL $2446 \mathrm{H} 8 \Omega$ compression driver, driven by a LABVIEW sine wave output and an external Pioneer A-107 audio amplifier. This was attached to a $460 \mathrm{~mm}$ long copper tube with a tube termination at the top (see Fig. 2). Interchangeable tube terminators were available with different radii of curvature $r$. Vacuum grease was applied to both contact regions to ensure a good seal. The resonant frequency was obtained using a microphone at the open end of the tube. The frequency applied to the loud speaker was adjusted until a maximum signal was obtained at the microphone. The resonant frequency was found to be $380 \mathrm{~Hz}$. A printed circuit board (PCB) Piezotronics $106 \mathrm{~B}$ pressure transducer was mounted on the wall of the copper tube of $160 \mathrm{~mm}$ from the open end (corresponding to the position of one of the microphones in Ref. 12). This was aligned with a $1 \mathrm{~mm}$ diameter hole in the copper tube and was used to monitor the sound pressure level (SPL) inside the tube. The acoustic particle velocity was found from the SPL using the expression for a plane traveling wave,

$$
S P L=20 \log _{10}\left(\frac{\rho_{0} c_{0}\left|u_{\mathrm{ac}}\right|}{\sqrt{2} p_{\mathrm{ref}}}\right),
$$

where $p_{\text {ref }}=2 \times 10^{-5} \mathrm{~Pa}$. 
A 20W Oxford Lasers LS20-50 copper vapor laser with a repetition rate of up to $50 \mathrm{kHz}$ was connected by an optical fiber to the fiberwire lightsheet optics which produced a lightsheet of $50 \mathrm{~mm}$ wide and of $1 \mathrm{~mm}$ thick at its narrowest point. The lightsheet was aligned with the axis of the copper tube using a translation stage and a PCO Sensicam camera was positioned perpendicular to the lightsheet. The system was sealed in a cubic aluminum box (painted matte black to reduce reflections) of length $1.2 \mathrm{~m}$, and seeding particles with a mean diameter of $1 \mu \mathrm{m}$ were introduced using a Safex Nebelgerät F2004 fog generator. The particles were introduced through a small sealable aperture and allowed to homogenize for at least 5 min prior to any measurement.

For each measurement, two images were captured by the CCD (charge-coupled device) camera. A Berkeley Nucleonics Corporation 500A pulse generator, which was triggered by the signal driving the speaker, controlled the time separation and exposure of the images by triggering both the camera and the laser pulses. The images were separated by $0.02 \mathrm{~ms}$ and were both exposed for $0.0004 \mathrm{~ms}$. The phase of the captured acoustic field was determined by the delay of the first exposure relative to the sinusoidal pulse driving the speaker. The two images were analyzed by dividing the images into interrogation regions of $32 \times 32$ pixels. A cross-correlation was performed between each interrogation region in the first image and the corresponding region in the second image. The displacement of the particles between images was found in each interrogation region from the position of the correlation peak. A three-point Gaussian fit $^{31}$ was applied at the peak to improve the accuracy of this procedure. Finally a local median filter was applied to remove outliers which differed from the local median value by more than a selected threshold. Outliers can be caused by random correlations due to high velocity gradients, low seeding density, or glare from surfaces.

\section{B. PIV measurement of velocity fields}

In this section velocity measurements obtained by PIV are presented for different SPLs representing potential flow and the two observed regimes: Formation of vortices at the open end which remain attached to the tube and vortex shedding from the open end. The velocity fields for a termination with $r=0$ (corresponding to $r<0.01 \mathrm{~mm}$ ) are shown in Figs. 3 and 4 for SPLs of 150, 160, and $170 \mathrm{~dB}$ (measured
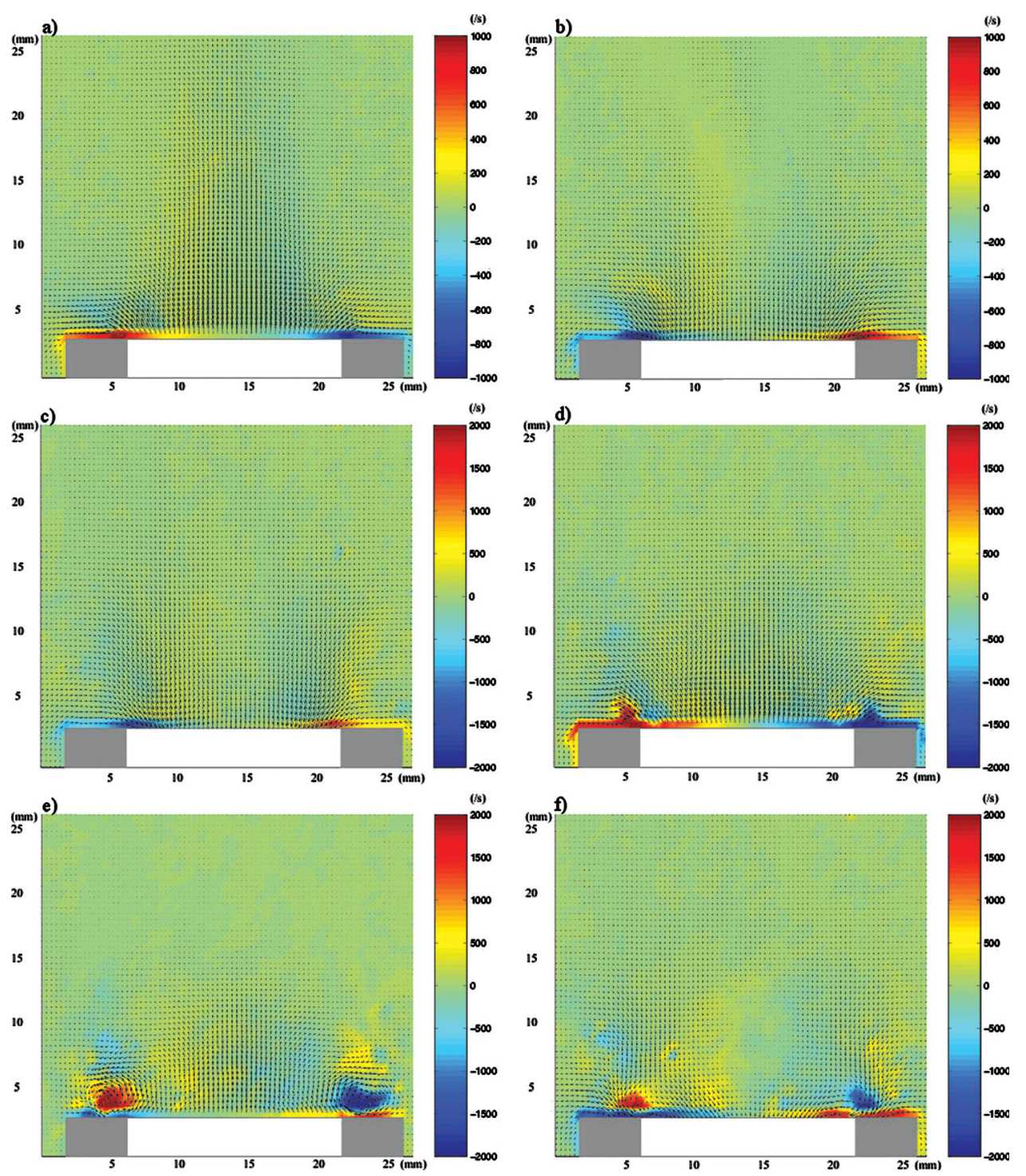

FIG. 3. (Color online) Velocity and vorticity fields at (a) $0.2 \mathrm{~T}$, (b) $0.8 \mathrm{~T}$ at a SPL of $150 \mathrm{~dB}$, (c) $0.0 \mathrm{~T}$, (d) 0.2 $T$, (e) $0.5 \mathrm{~T}$, and (f) $0.6 \mathrm{~T}$ at a SPL of $160 \mathrm{~dB}$, for $r=0$. 

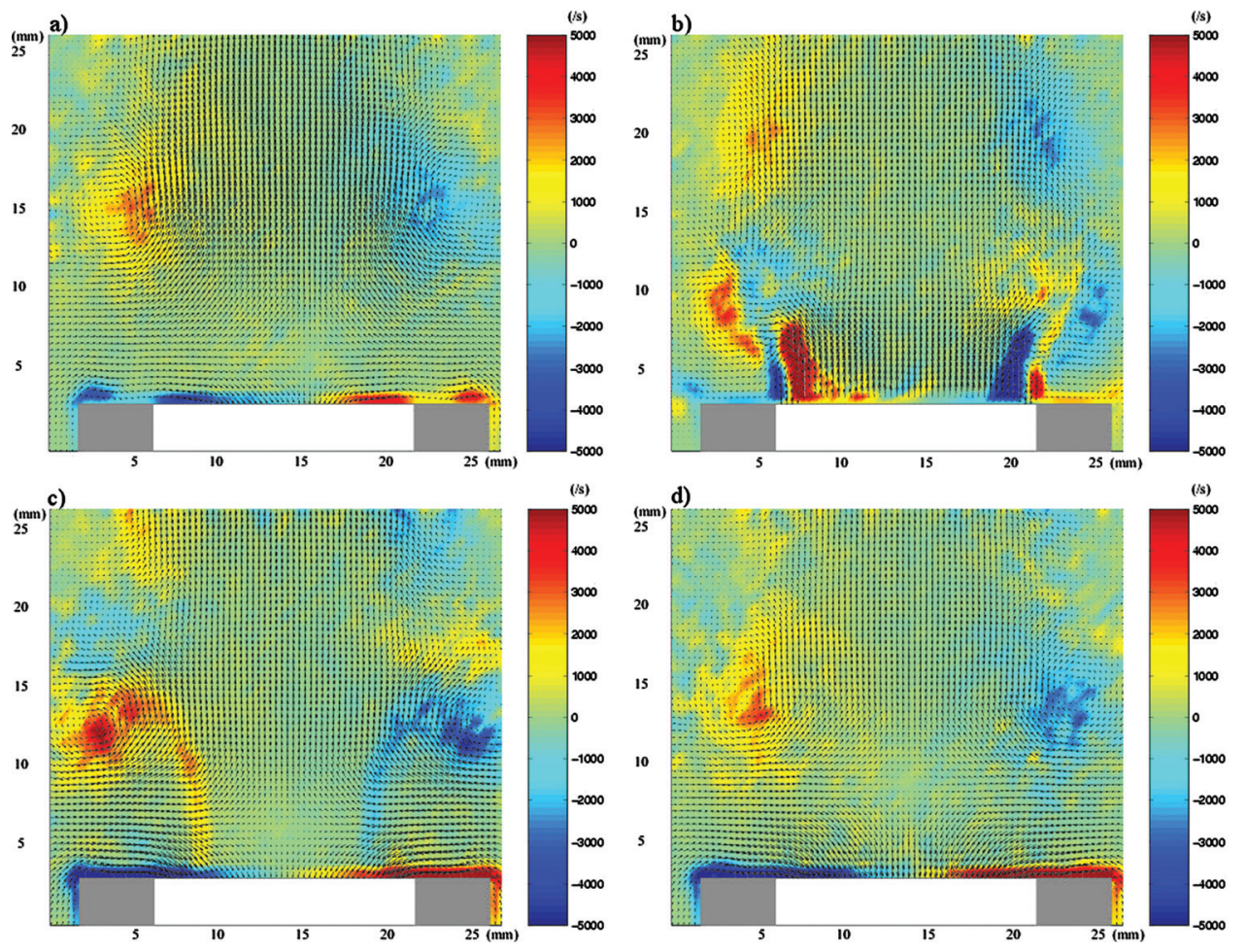

FIG. 4. (Color online) Velocity and vorticity fields at (a) $0.0 \mathrm{~T}$, (b) $0.3 \mathrm{~T}$, (c) $0.6 \mathrm{~T}$, and (d) $0.9 \mathrm{~T}$ at a SPL of $170 \mathrm{~dB}$ for $r=0$. using the pressure transducer on the wall of the tube), respectively.

Figures 3(a) and 3(b) indicate the velocity field at $0.2 T$ and $0.8 T$ for a SPL of $150 \mathrm{~dB}$. The velocity fields in Figs. 3(a) and 3(b) are approximately potential with very little vorticity present. This was observed in all the result for $r=0 \mathrm{~mm}$ at this sound level. Zones of vorticity are present on the edge of the tube in Figs. 3(a) and 3(b) and in all the other PIV images presented. At high Strouhal numbers it is expected that the vorticity remains close to the edges of the tube and it is possible that these regions of vorticity may be physically correct. However, given there position close to the surface of the tube, it is also possible that they result from reflections of the laser light on the surface of the tube. It is therefore not possible to say with certainty that these small zones of vorticity are a real feature of the flow.

At a SPL of $160 \mathrm{~dB}$ [Figs. 3(c)-3(f)] two regions of vorticity are observed close to the open end of the tube. These are developed when the velocity is directed out of the tube and slowly diminish after flow reversal. The two regions of vorticity correspond to a $2-\mathrm{D}$ slice through a 3 -D swirling ring which remains attached to the top of the tube.

Regions of vorticity are also produced at a SPL of 170 dB (see Fig. 4). A permanent jet directed away from the tube was also observed at this SPL. This is in agreement with the measurements of Tartarin ${ }^{34}$ taken several diameters from the tube, and those of Peube ${ }^{35}$ who observed a jet for a Reynolds number based on the acoustic boundary layer thickness $R e_{\delta}=A \sqrt{f_{0} / v} /(2 \pi)>22$, where $A$ is the acoustic displacement amplitude and $f_{0}$ is the fundamental frequency. Here $R e_{\delta} \simeq 35$ at $170 \mathrm{~dB}$. The vorticity is shed and convects with a velocity $v_{\Gamma} \simeq \hat{v}_{j} / 4$, where $\hat{v}_{j}$ is the velocity amplitude of the jet.
The mechanism behind the formation of this jet is evident in Fig. 4. During outflow from the open end, the velocity is largest in the central region initiating the jet. At flow reversal the circulating vortex ring above the opening entrains the surrounding air and draws it in from the sides. This air flow feeds into the central jet. As the suction stage of the acoustic cycle progresses the air drawn in from the sides is drawn into the tube more strongly at the sides of the tube and the outward jet remains present. A stagnation point is present just outside the open end during outflow. A fuller description of this flow is presented elsewhere. ${ }^{36}$

PIV measurements for $r=0.3 \mathrm{~mm}$ and $r=1 \mathrm{~mm}$ showed a number of similarities to those observed for the $r=0 \mathrm{~mm}$ termination, with transition between the regimes occurring at a higher SPL. In particular, the transition to vortex shedding was observed at an SPL of around $166.7 \mathrm{~dB}$ $\left(u_{\mathrm{ac}}=14.8 \mathrm{~ms}^{-1}\right)$ for $r=0.3 \mathrm{~mm}$ and at around $169.1 \mathrm{~dB}$ $\left(u_{\mathrm{ac}}=19.33 \mathrm{~ms}^{-1}\right)$ for $r=1 \mathrm{~mm}$.

\section{Determination of losses due to the interaction between vortices formed at the opening and the radiated acoustic field}

The real part of the termination impedance is given by Eqs. (15) and (19) in terms of the continuous fluid velocity $\boldsymbol{v}$, vorticity $\Omega$, and acoustic particle velocity $\mathbf{u}_{\mathrm{ac}}$. To perform this calculation using the PIV measurements, each of these three quantities must be extracted form the data and the discrete and planar nature of the measurements must be accounted for. The velocity field was assumed to be axi-symmetric and the data set was reduced by taking the average of points on symmetrically opposite sides of the tube. The velocity points were then labeled by the index $i=1,2$, 
$3, \ldots, n$ in the direction away from the tube axis and the index $j=1,2, \ldots, m$ in the axial direction. This gives the discrete velocity field $\boldsymbol{v}(i, j)$. The discrete viscosity field $\boldsymbol{\Omega}(i, j)$ was found using the curl function in Matlab. At $150 \mathrm{~dB}$ the discrete acoustic particle velocity, $\boldsymbol{u}_{\mathrm{ac}}(i, j)$, was taken to be the total velocity field since it was approximately potential. At higher SPL, the acoustic particle velocity was found from the velocity field at $150 \mathrm{~dB}$ scaled to account for the increased intensity. Thus at a sound pressure level of $x \mathrm{~dB}$, the acoustic particle velocity was taken to be the measured velocity field at $150 \mathrm{~dB}$ with the magnitude of the velocities scaled by a factor $10^{(x-150) / 20}$.

Equation (15) was then be re-written in a discrete form as

$$
\mathcal{P}_{s}=-\sum_{i, j}[\Omega(i, j) \times \boldsymbol{v}(i, j)] \cdot u_{\mathrm{ac}}(i, j) M(i, j) .
$$

If the separation between data points in the axial and radial direction is $\Delta x$, then $M(i, j)$, the mass of fluid within the measurement volume containing $(i, j)$, is given by the product of the density, the separation between discrete points in the axial direction, and the difference is area between two concentric circles of radii $i \Delta x$ and $(i-1) \Delta x$,

$$
M(i, j)=(2 i-1) \rho_{0} \pi(\Delta x)^{3}
$$

The time-average was also performed in a discrete manner by averaging over ten equally spaced measurements throughout the period. The termination impedance results for $r=0 \mathrm{~mm}$ are shown in Fig. 5 for a range of SPL corresponding to the acoustic particle velocity at the end of the tube, $u_{a c}$, varying from around $5 \mathrm{~ms}^{-1}$ to around $17 \mathrm{~ms}^{-1}$. They show an increase in the real component of the termination impedance with the acoustic particle velocity at the open end of the tube, up to around $9 \mathrm{~ms}^{-1}$. There is also evidence of a discontinuity at an acoustic particle velocity between 9 and $16 \mathrm{~ms}^{-1}$. Measurements were also taken at $r=4 \mathrm{~mm}$, however no vorticity was observed at any of the SPLs considered.

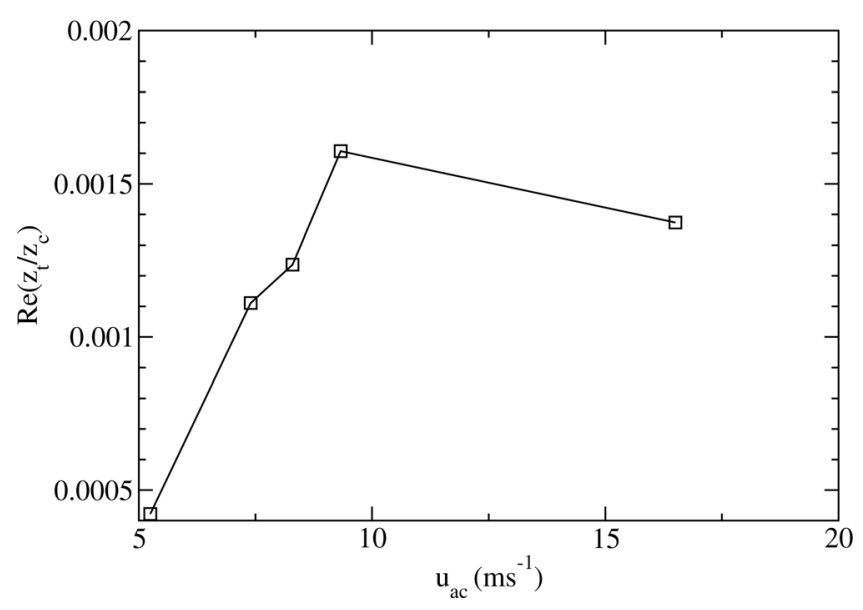

FIG. 5. The real part of the termination impedance calculated from the PIV velocity measurements obtained outside the tube.

\section{LBM SIMULATION OF THE ACOUSTIC, VELOCITY AND VORTICITY FIELDS AT THE OPEN END OF A RESONATING TUBE}

The lattice Boltzmann method (LBM) is a numerical technique which has been developed primarily to simulate fluid flow. ${ }^{37}$ It has also been shown to be suitable for simulating acoustic waves at low Mach number. ${ }^{38-46}$ A brief description of the LBM relevant to the simulations presented here is given below. A wider discussion of the LBM is available in Refs. 37, 47, and 48.

\section{A. The LBM}

In the LBM a simulation is performed on a regular grid where each grid point, or node, is connected to $i$ that neighbors along the link directions $\mathbf{e}_{i}$. At each node, $x$, and each discrete time-step, $t$, there is a distribution function, $f_{i}(x, t)$, associated with all of the link directions $\mathbf{e}_{i}$. The LBM describes a fluid by simulating the evolution of these distribution functions. Here the D2Q9 lattice is applied. This uses a 2-D grid with nine link directions: $\mathbf{e}_{0}=(0,0), \mathbf{e}_{1}=(1,0)$, $\mathbf{e}_{2}=(0,1), \mathbf{e}_{3}=(-1,0), \mathbf{e}_{4}=(0,-1), \mathbf{e}_{5}=(1,1), \mathbf{e}_{6}=(-1,1)$, $\mathbf{e}_{7}=(-1,-1)$, and $\mathbf{e}_{8}=(1,-1)$. The distribution functions evolve according to the Boltzmann equation, ${ }^{49}$

$$
f_{i}\left(\boldsymbol{x}+\mathbf{e}_{i}, t+1\right)-f_{i}(\boldsymbol{x}, t)=-\frac{1}{\tau}\left(f_{i}-\bar{f}_{i}\right),
$$

where the relaxation time, $\tau$ is found from the kinematic viscosity $v$ of the fluid,

$$
v=\frac{2 \tau-1}{6}
$$

The left hand side of Eq. (23) represents streaming of the distribution function from one site to the neighboring site. The right hand side is the Bhatnagar, Gross, and Krook (BGK) collision operator ${ }^{50-52}$ which mimics collisions between distribution functions as a relaxation toward the equilibrium distribution function $\bar{f}_{i}$. The form of this function is described below.

The physical parameters of interest are the fluid velocity and pressure. These are obtained from the distribution functions and through the equation of $\operatorname{state}^{37} p=c_{0}^{2} \rho$ and using

$$
\rho=\sum_{i=0}^{8} f_{i} \quad \text { and } \quad \rho \boldsymbol{v}=\sum_{i=0}^{8} f_{i} \mathbf{e}_{i} .
$$

The equilibrium distribution function $\bar{f}_{i}$ is given by ${ }^{49}$

$$
\overline{f_{i}}=w_{i} \rho\left[1+3 \mathbf{e}_{i} \cdot \boldsymbol{v}+\frac{9}{2}\left(\mathbf{e}_{i} \cdot \boldsymbol{v}\right)^{2}-\frac{3}{2} \boldsymbol{v}^{2}\right],
$$

where the weight functions are given by $w_{0}=4 / 9, w_{i}=1 / 9$ for $i=1-4$, and $w_{i}=1 / 36$ for $i=5-8$.

The Navier-Stokes equation can be obtained from Eq. (23) by performing a Chapman-Enskog expansion. ${ }^{37,53}$ This verifies that the LBM mimics a real fluid in which $c_{0}=\sqrt{\frac{1}{3}}$. 


\section{B. LBM simulations of velocity fields: Simulation domain}

In this section the velocity fields around the open end of a resonating tube are presented. These were performed using the 2D LBM described in Sec. V A. The computational grid is shown in Fig. 6. The dotted line represents an anechoic boundary condition, where the unknown distribution functions were determined by a backward linear interpolation. ${ }^{54,55}$ This boundary condition absorbs any flow or acoustic signal at the boundary without introducing a reflection. Thus the computational boundary has a negligible effect on the simulated flow. The thick solid line represents an acoustic source. At this boundary the distribution functions were set equal to their equilibrium values ${ }^{38}$ for the required velocity distribution, in this case a sinusoidally varying velocity. The thin solid line represents a no-slip boundary. The no-slip boundary was implemented using an interpolation scheme ${ }^{56}$ which simulated the position of the boundary to sub-grid accuracy while maintaining the second-order accuracy of the LBM. This scheme ensured that the radius of curvature of the tube opening was simulated accurately and not by a step-like approximation with the resolution of the grid.

\section{LBM simulation of velocity fields: Results}

At low acoustic particle velocities the flow was found to be completely potential. Two regimes were observed in the non-linear case. At moderate acoustic particle velocities, vorticity was observed at the opening of the tube. This is shown in Fig. 7 for a radius of curvature $r=0 \mathrm{~mm}$.

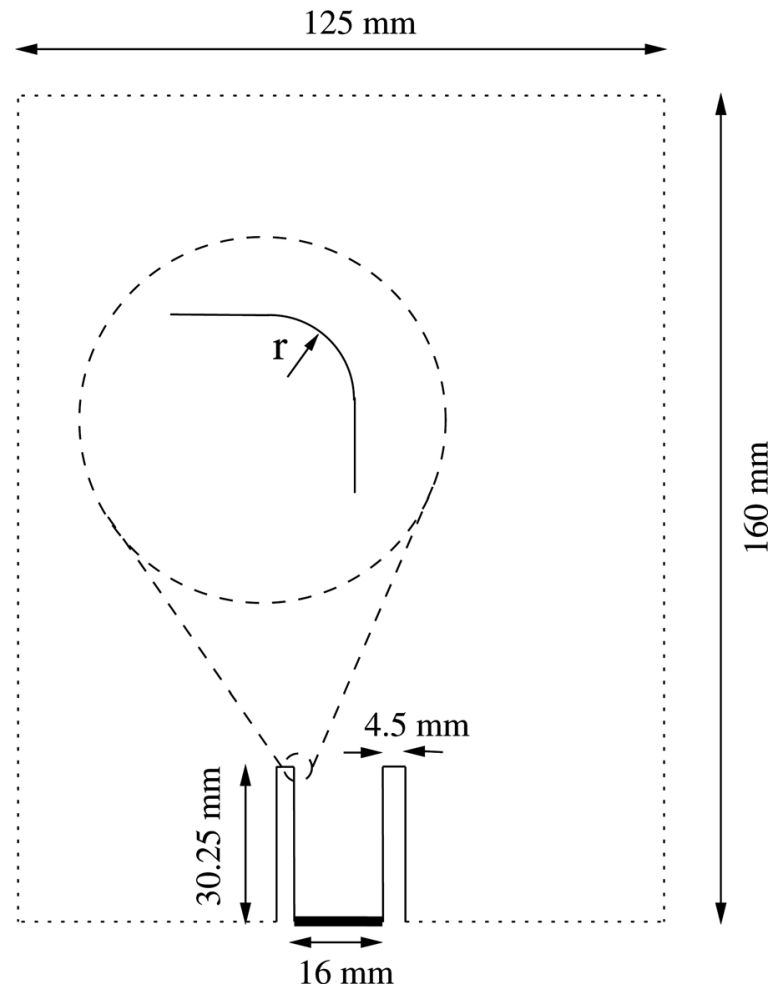

FIG. 6. The 2-D computational grid used for the LBM simulations. The boundary conditions are represented by a dotted line (anechoic), a thin solid line (no-slip), and a thick solid line (acoustic source).

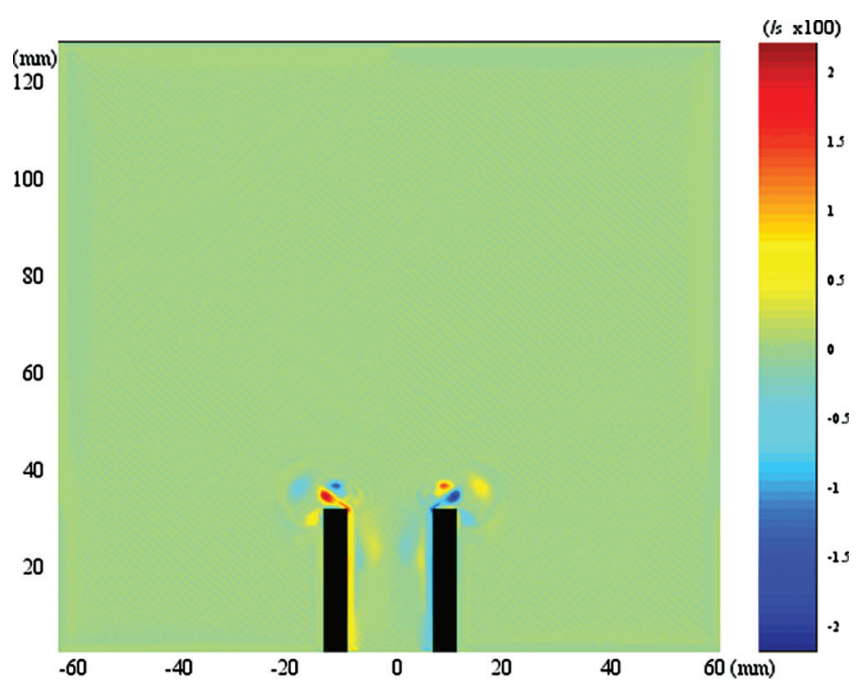

FIG. 7. (Color online) Vorticity field at a moderate acoustic particle velocity $\left(10 \mathrm{~ms}^{-1}\right)$ for a frequency of $f_{0}=380 \mathrm{~Hz}$.

The vorticity was seen to remain at the open end of the tube in a similar manner to that observed using PIV, shown in Figs. 3(c)-3(f). The second non-linear regime occurred at higher acoustic particle velocities where vortex shedding was observed (see Fig. 8). The formation of a pair of vortices at the open end of the tube is shown in Fig. 8(a). The vorticity is then shed and moves away at an angle of approximately $45^{\circ}$, as a new pair of vortices is generated. The LBM simulated velocity fields show the inside of the tube where the formation of vorticity is observed in Figs. 8(c) and 8(d). There are significant differences between the vortex behavior observed in the LBM simulations and the PIV measurements. In particular, due to the 2D nature of the LBM simulations, the vortices observed above the left and right hand walls of the tube are independent of each other. This is in contrast to the PIV measurements which show a cross-section through a vortex ring, and the two observed "vortices" are in fact part of the same larger structure. It is therefore not possible to compare the vorticity, or indeed the velocity fields. It is, nevertheless, evident from the LBM simulations that there are two regimes in which vortex motion is present: the first at a lower acoustic particle velocity where the vortex remains attached to the wall and a second at a higher acoustic particle velocity where the vortex becomes detached. In the following section the losses associated with the interaction between the vortices and the acoustic field will be established to investigate whether the change in the vortex shedding regime is associated with the discontinuity in the termination impedance observed by Atig et al. ${ }^{12}$ Given the limitations of the LBM simulations, it will not be possible to determine quantitative values for the termination impedance so the results can not be directly compared to those obtained from the PIV measurements or previous studies. ${ }^{12}$ The aim here, however, is to investigate whether a transition between the two observed regimes of vortex shedding can be related to a change in the termination impedance and explain the measurements of Atig et al. ${ }^{12}$ 

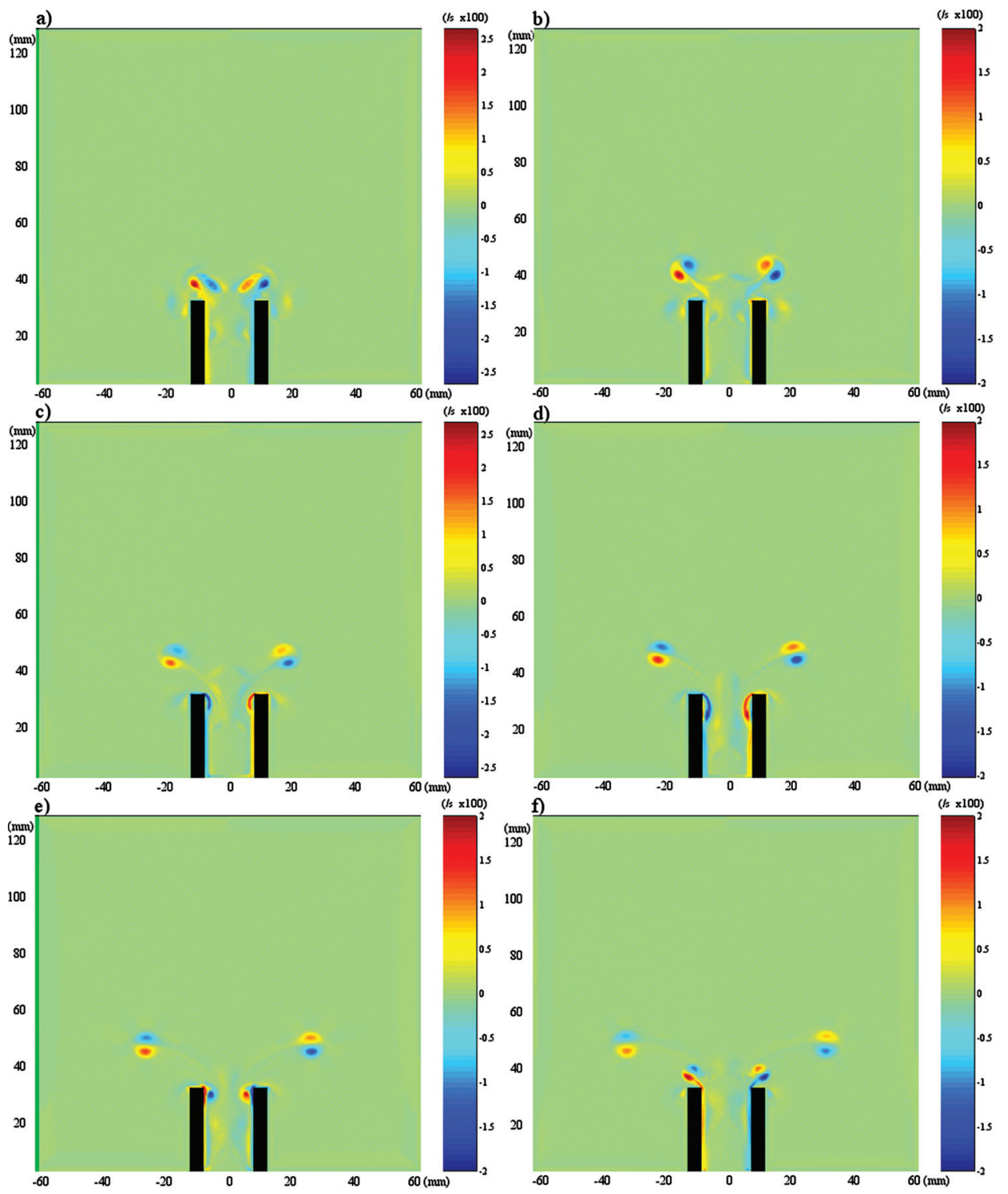

FIG. 8. (Color online) Vorticity field at a moderate acoustic particle velocity $\left(27 \mathrm{~ms}^{-1}\right)$ for a frequency of $f_{0}=380 \mathrm{~Hz}$ at (a) $0.0 \mathrm{~T}$, (b) 0.2 $T$, (c) $0.4 T$, (d) $0.6 T$, (e) $0.8 T$ and (f) $1.0 T$.

\section{Determination of losses due to the interaction between vortices formed at the opening and the radiated acoustic field}

In this section, the losses associated with the interaction between the acoustic field and the vortices formed at the open end of the resonating tube are considered using the velocity information from the LBM simulations. The real part of the terminal impedance was calculated in a similar manner to that used for the PIV measurements. It was possible to perform a simulation at a very low intensity without introducing errors due to resolution or noise issues so an acoustic particle velocity of $10^{-3} \mathrm{~ms}^{-1}$ was chosen for the "potential flow" simulation. Due to the 2D nature of the simulations the definition of the mass of the simulation volume was taken to be

$$
M(i, j)=(2 i-1) \rho_{0} \pi(\Delta x)^{2}
$$

It was found that the losses were not completely repeatable over each period. To overcome this, the results were averaged over the final five periods of the simulation. Averaged termination impedances are shown in Fig. 9 for $r=0 \mathrm{~mm}$ and $r=4 \mathrm{~mm}$. The solid line was obtained by integrating over the exterior of the tube and the dashed line by including the interior of the tube in the calculation. A clear transition in the profile of the real part of the termination impedance curve can be seen in Fig. 9 at acoustic particle velocities of around $20 \mathrm{~ms}^{-1}$ for $r=0 \mathrm{~mm}$ and $27 \mathrm{~ms}^{-1}$ for $r=4 \mathrm{~mm}$. It is also evident that losses are occurring at both inside and outside the tube. The majority of the losses occurred outside the tube, with the losses occurred inside the tube was accounting for approximately $20 \%$ of the total.

\section{THEORETICAL MODEL FOR ENERGY LOSSES DUE TO THE INTERACTION BETWEEN VORTICES FORMED AT THE OPENING AND THE RADIATED ACOUSTIC FIELD}

In this section a theoretical model is considered for the acoustic particle velocity, the fluid velocity, the regions of vorticity, and the losses associated with the interaction between the acoustic particle velocity field and the vortices. The aim is to provide a qualitative understanding of the 


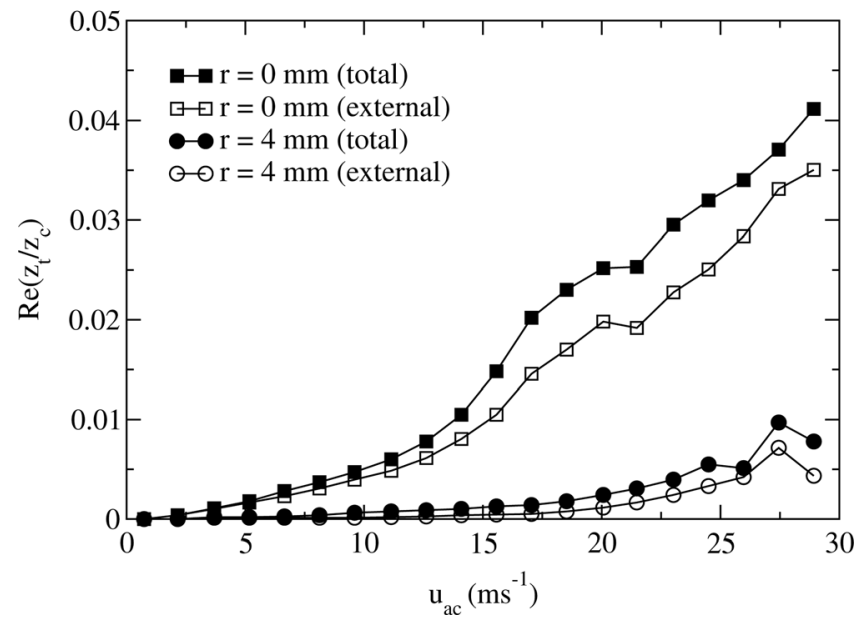

FIG. 9. Real part of the termination impedance as a function of the acoustic particle velocity calculated from the LBM velocity fields. The open symbols were calculated using only points external to the tube; the filled symbols were calculated using both external and internal points.

problem using a simplified model, following the approach of Hirschberg et al. ${ }^{57}$ and based on the velocity fields obtained from the LBM simulations and PIV measurements presented here and in Refs. 36 and 29.

In Secs. IV and V two distinct non-linear regimes were observed. At low acoustic particle velocity, the linear case, the flow was a good approximation to potential flow. At moderate acoustic particle velocities the first non-linear regime was observed: Vortex structures were generated during outflow, which remained attached to the walls at the open end and were sucked into the tube during inflow. At high acoustic levels a jet was observed close to the opening of the tube and the second non-linear regime was observed. Here the vortex structures were shed and their motion was influenced by a fluid jet directed out of the open end of the tube. Vortex pairs were observed which were formed during outflow and also inside the tube during inflow and subsequently expelled during outflow. The actual SPL at which the transition between these regimes occurred was dependent on the geometry of the termination.

In the theoretical model the sound field was approximated by the analytical expression for the acoustic particle velocity from a monopolar diaphragm source at the center of the termination in a tube with walls of zero thickness, ${ }^{22}$

$$
\mathbf{u}_{\mathrm{ac}}=\frac{Q}{2 \pi\left[r^{2}+z^{2}\right]^{3 / 2}} \sin \left(\omega_{0} t\right)\left(r \mathbf{e}_{r}+z \mathbf{e}_{z}\right),
$$

where $Q=\pi a^{2} \hat{u}_{s}$, and $\hat{u}_{s}$ is the desired amplitude of the acoustic field at the open end of the tube

$$
u_{s}(t)=\hat{u}_{s} \sin \left(\omega_{0} t\right) \text {. }
$$

Equations (28) and (29) are sufficient to model the behavior observed in the linear potential flow case. The jet formation observed during outflow $(0<t<T / 2)$ in the two non-linear regimes was incorporated into the model by defining the velocity as

$$
\boldsymbol{v}= \begin{cases}\hat{u}_{s}\left(\frac{a}{r_{j}}\right)^{2} \sin \left(\omega_{0} t\right) \mathbf{e}_{z} & \left|e_{r}\right|<r_{j}, 0<t<T / 2 \\ 0 & \left|e_{r}\right|>t_{j}, 0<t<T / 2 \\ \mathbf{u}_{\mathrm{ac}} & T / 2<t<T,\end{cases}
$$

where $r_{j}$ is the radius of the jet, and $\boldsymbol{v}_{j}=\hat{\boldsymbol{v}}_{j} \sin \left(\omega_{0} t\right) \mathbf{e}_{z}$ is the velocity determined by continuity: $\pi a^{2} u_{s}=\pi r_{j}^{2} v_{j}$. This is shown schematically in Fig. 10.

Observations in the two non-linear regimes indicated a region of vorticity outside the tube, which developed during the first half period and then diminished during the second half. To incorporate this, the vorticity model was developed following Nelson et al. ${ }^{58}$ Consider the contour path of length $\Delta_{s}$ in the $\mathrm{e}_{z}$ direction shown in Fig. 10. The circulation around this contour was expressed, using Stoke's theorem, as

$$
\Delta \Gamma=\oint_{C} v \cdot \mathrm{d} l=-v_{j} \Delta s=\iint_{S} \Omega \cdot \mathrm{d} \mathbf{S} .
$$

Fluid inside the contour path was assumed to be convected with speed $v_{c}=v_{j} / 2$, the mean of the speed inside and outside the jet. Thus

$$
\frac{\mathrm{d} \Gamma}{\mathrm{d} t}=\frac{\mathrm{d} \Gamma}{\mathrm{d} s} \frac{\mathrm{d} s}{\mathrm{~d} t}=-\boldsymbol{v}_{j} \boldsymbol{v}_{c}
$$

From Eq. (15), with $\langle\mathcal{P}\rangle=-\left\langle\mathcal{P}_{s}\right\rangle$ since only dissipation outside the tube is considered,

$$
\langle\mathcal{P}\rangle=\rho_{0} \frac{1}{T} \int_{0}^{T} \int_{V}(\Omega \times \boldsymbol{v}) \cdot \mathbf{u}_{\mathrm{ac}} \mathrm{d} V \mathrm{~d} t .
$$

For $T / 2<t<T, \boldsymbol{v}$ is equal to $\mathbf{u}_{\mathrm{ac}}$ and the integrand is identically zero. By symmetry $\mathrm{d} V=2 \pi r \mathrm{~d} r \mathrm{~d} z$. If the circulation is considered to be at position $\left(r_{\Gamma}, z_{\Gamma}\right)$ (see Fig. 10), then $\Omega$ $=\Gamma \delta\left(r-r_{\Gamma}\right) \delta\left(z \Gamma z_{\Gamma}\right) \mathbf{e}_{\theta}$; for $0<t<T / 2$, where $\Gamma$ is obtained by integrating Eq. (32): $\Gamma=-\hat{v}_{j} \hat{v}_{c} \int_{0}^{t} \sin _{2}\left(\omega_{0} t\right)$. This gives a final expression
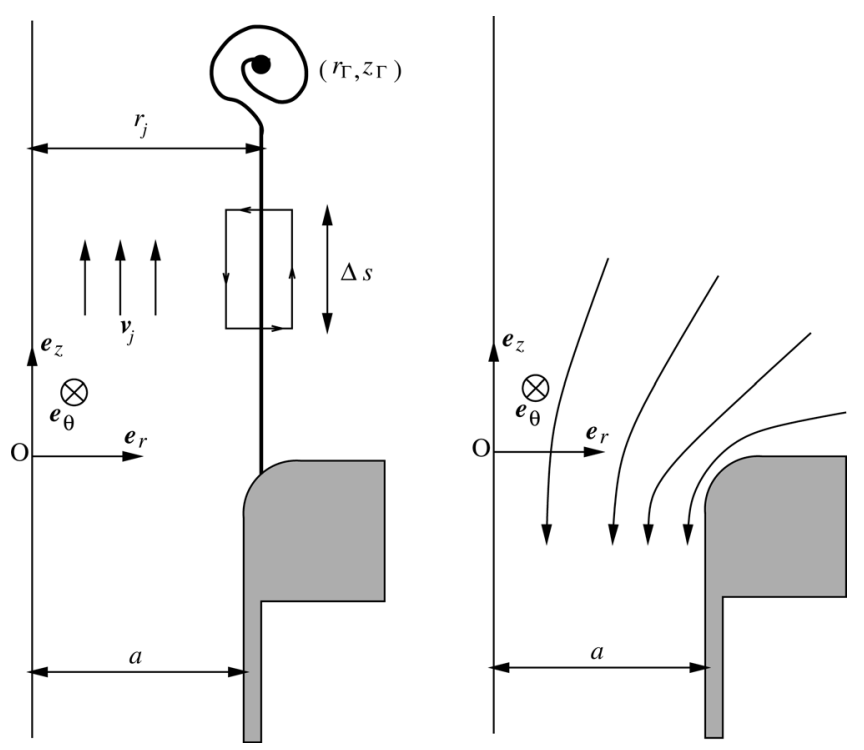

FIG. 10. A schematic diagram indicating the parameters for the physical model. 


$$
\begin{aligned}
\langle\mathcal{P}\rangle= & \frac{\rho_{0} \pi a^{2} \hat{u}_{s}^{4}}{2}\left(\frac{a}{r_{j}}\right)^{6} \frac{1}{T} \\
& \times \int_{0}^{T / 2} \frac{r_{\Gamma}^{2}}{\left(r_{\Gamma}^{2}+z_{\Gamma}^{2}\right)^{3 / 2}}\left[\frac{t}{2}-\frac{\sin \left(2 \omega_{0} t\right)}{4 \omega_{0}}\right] \\
& \times \sin ^{2}\left(\omega_{0} t\right) d t .
\end{aligned}
$$

\section{A. Application of the theoretical model}

Observations identified two regimes where vorticity was present: one at moderate acoustic amplitudes where the vortex remained attached to the termination and a second at higher amplitudes where the vortex is separated. The application of the model to these regimes will be considered separately.

\section{Attached vortex}

When the vortex remains attached to the termination, its coordinates $\left(r_{\Gamma}, z_{\Gamma}\right)$ were considered to be constant. Combining Eqs. (19) and (34) we obtain

$$
\Re\left(\frac{Z_{t}}{Z_{c}}\right)=\frac{2 \mathcal{P}}{\pi a^{2} \hat{u}_{s}^{2} Z_{c}}=\frac{\hat{u}_{s}^{2}}{32 f_{0} c_{0}}\left(\frac{a}{r_{j}}\right)^{6} \frac{r_{\Gamma}^{2}}{\left(r_{\Gamma}^{2}+z_{\Gamma}^{2}\right)^{3 / 2}} .
$$

For $r=0$, the position of the clinging vortex was estimated from the PIV images to be $\left(r_{\Gamma}=a, z_{\Gamma}=a / 3\right)$ corresponding to the edge of the jet $\left(r_{j}=r_{\Gamma}\right)$. For $r>0$ it was assumed that $r_{\Gamma}=a+r, z_{\Gamma}=a / 3$, and $r_{j}=r_{\Gamma}$. The termination impedance calculated from the attached vortex model is shown in Fig. 11(a) for four values of $r$. The results of the attached vortex model indicate an increase in the losses with increased acoustic particle velocity. They also show that the losses are very sensitive to the radius of curvature of the open end; the largest losses occurring for $r=0$.

\section{Convecting vortex}

At higher acoustic particle velocities the vortex was seen to move away from the termination. In this case it is assumed that $r_{\Gamma}=a+r, r_{j}=r_{\Gamma}$, and $z_{\Gamma}=a / 3+\hat{\boldsymbol{v}}_{\Gamma} t$, such that Eq. (34) becomes

$$
\begin{aligned}
\langle\mathcal{P}\rangle= & \frac{\rho_{0} \pi a^{2} \hat{u}_{s}^{4} r_{\Gamma}^{2}}{2}\left(\frac{a}{r_{j}}\right)^{6} \frac{1}{T} \\
& \times \int_{0}^{T / 2} \frac{1}{\left(r_{\Gamma}^{2}+z_{\Gamma}^{2}(t)\right)^{3 / 2}}\left[\frac{t}{2}-\frac{\sin \left(2 \omega_{0} t\right)}{4 \omega_{0}}\right] \\
& \times \sin ^{2}\left(\omega_{0} t\right) d t .
\end{aligned}
$$

From the PIV observations, $\hat{v}_{\Gamma}$ was taken to be $\hat{\boldsymbol{v}}_{j} / 4$ and the results of numerically integrating Eq. (36) are shown in Fig. 11(b). The termination impedance results for the convecting vortex model are similar to that of the fixed vortex model at low acoustic particle velocities. At higher acoustic particle velocities the convecting vortex model predicts losses which increase in a quasi-linear manner with increasing acoustic particle velocity. The model also predicts losses which are sensitive to the radius of curvature at the open end.

\section{B. Transition between the regimes}

The termination impedance for both the attached and the convecting models is shown in Fig. 12 for $r=0$. For acoustic particle velocities less than about $15 \mathrm{~ms}^{-1}$, the difference between the models is small. At higher speeds the fixed model for the termination impedance continues to display a quadratic-like dependence, while the convecting model is quasi-linear. The regime change observed in Ref. 12 can be understood by the transition from the attached to the convecting vortex models occurring at an acoustic particle velocity where shedding is triggered. This is illustrated in Fig. 12 by the symbols.

\section{DISCUSSION AND CONCLUSION}

The acoustic energy losses at the end of a tube have been investigated using different approaches. PIV observation showed that annular vortices are generated at the end of tube, which validates previous interpretations. ${ }^{10,11}$ Moreover, PIV experiments indicated that two different regimes can occur: one for which the annular vortices remain attached to the tube (low acoustic particle velocity) and one for which the annular vortices become detached and leave the tube (high acoustic particle velocity). Application of vortex-sound theory to the PIV measurements showed a regime change similar to that observed by Atig et al. ${ }^{12}$ It was seen that the transition between the two regimes for the termination impedance occurred at an acoustic particle velocity comparable to that at which the vortices started to detach from the tube. There were a number of physical differences between the PIV measurements and the 2D LBM simulations, not least in the nature of the vortices formed at the open end of the tube, and the manner in which they were shed. Despite these clear differences the termination impedance calculated from the LBM simulations also showed a
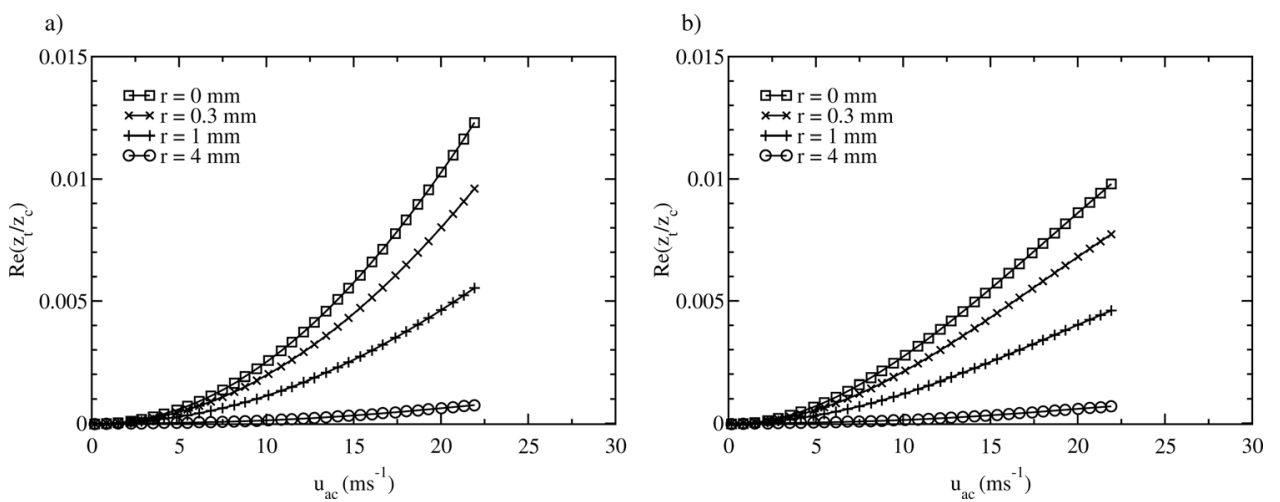

FIG. 11. Influence of the curvature $r$ on the termination impedance for (a) the attached vortex model and (b) the convecting vortex model. 


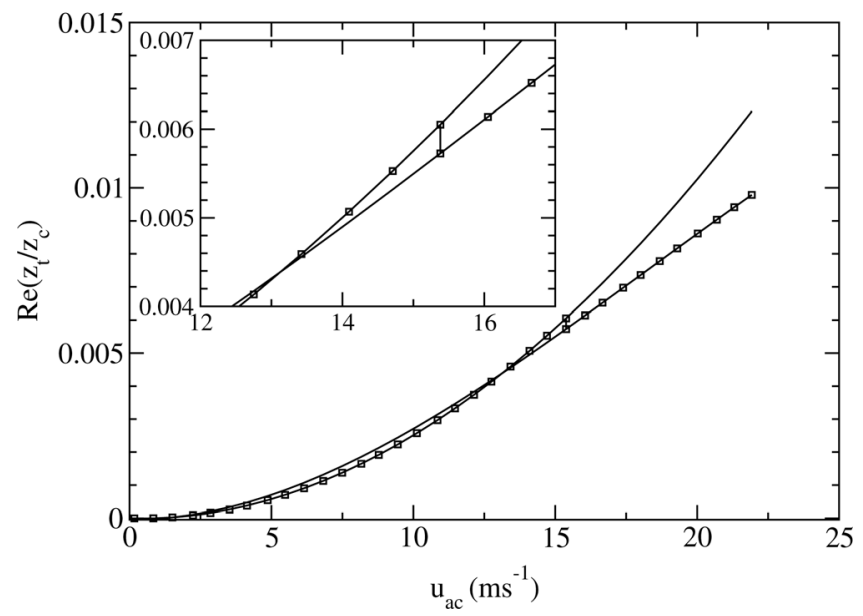

FIG. 12. The termination impedance for both the attached (--*-) and the convecting (-) vortex models for $r=0$. The symbols and the solid line represent a possible transition between the two models.

distinct change between two regimes which was again associated with a change from the vortices being attached to the open end of the tube and the vortices being shed. The analytical model provided two different laws relating to the losses and the acoustic particle velocity: one for an attached vortex and one with a detached vortex. A transition between these two models occurs at the acoustic particle velocity associated with the onset of vortex shedding.

PIV measurements, LBM simulation, and the analytical model all failed to predict the exact magnitude of the nonlinear losses. The analytical model is too simple to consider all the losses and to predict the acoustic particle velocity at which the transition occurs. The fact that the LBM is only 2-D does not allow for more than the qualitative results. Analysis of the PIV measurements gave a consistent acoustic particle velocity for the transition but underestimated the magnitude of the losses, compared to the measurements of Atig et al. ${ }^{12}$ The accuracy of the PIV measurements depends on a number of factors such as the size and concentration of the seeding particle, the size of the interrogation region relative to the velocity gradients, and particle motion in or out of the light plane. ${ }^{24,31}$ Two of the most significant sources of error are due to the accuracy with which the correlation peaks can be determined in the analysis procedure and the assumption that the seeding particle accurately follow the flow. Here a Gaussian three-point fit was applied, ${ }^{31}$ which enables the displacement to be found to sub-pixel accuracy of between \pm 0.05 and \pm 0.1 pixels. ${ }^{31}$ The motion of the seeding particle will deviate from that of the air because of weight and buoyancy forces, the radiation force due to the acoustic field, and an impeded response to any fluid acceleration due to the inertia of the particle. Here the seeding particles fog had a mean particle diameter of only $1 \mu \mathrm{m}$ and no measurable motion due to their weight was observed. Indeed, the particles were allowed to homogenize for at least 5 min before any measurements were taken and they were not observed to settle during this time. Following Buick et al. ${ }^{59}$ the velocity due to the radiation force ${ }^{60} u_{\text {rad }}$ of a seeding particle of radius $a_{s p}$ and relative density $\Lambda$ in a progressive acoustic wave is

$$
u_{\mathrm{rad}}=\frac{P^{2} k^{6} a_{\mathrm{sp}}^{5}}{6 \pi v \rho_{0}^{2} f^{2}} \frac{\left[1+2(1-\Lambda]^{2} / 6\right]}{(2+\Lambda)^{2}} .
$$

For the maximum pressure, $P$, corresponding to an SPL of $170 \mathrm{~dB}$, this gives a negligible velocity of around $10^{-20} \mathrm{~ms}^{-1}$. Vignola et al. ${ }^{61}$ showed that the inertia effect can be expressed in terms of the relaxation time

$$
\tau_{p}=\frac{4 a_{\mathrm{sp}}^{2} \Lambda}{18 v}
$$

for a seeding particle in an acoustic field. The acoustic particle velocity $u_{\mathrm{ac}}$ and the seeding particle velocity $u_{\mathrm{sp}}$ are then related through $\chi=2 \pi f \tau_{p}{ }^{62}$

$$
\begin{aligned}
& \left|u_{\mathrm{ac}}\right|=\left|u_{\mathrm{sp}}\right| \sqrt{1+\chi} \\
& \angle u_{\mathrm{ac}}=\angle u_{\mathrm{sp}}+\tan ^{-1}(\chi) .
\end{aligned}
$$

Here $\sqrt{1+\chi}=1.004$ and $\tan ^{-1}(\chi)=0.5^{\circ}$ and are both negligible.

The relatively small errors associated with PIV do not, therefore, account for the larger differences between the termination impedance determined from the PIV measurements and the existing data ${ }^{12}$ obtained using the two microphone method in the tube. The discrepancy in the magnitudes of the losses can be partially explained by losses occurring outside the measurement region. Additionally only the losses due to the interaction of the vortices with the acoustic field were considered. Clearly other sources of energy loss, such as the formation of the vortices, were also present.

In conclusion, the losses due to the interaction of vortices formed at the open end of a resonating tube and the radiated acoustic field have been investigated using PIV measurement, LBM simulation, and an analytical model. Each approach has identified two regimes: the first occurring at lower acoustic particle velocities when the vortices remain attached to the open end of the pipe. The second occurs as higher acoustic particle velocities and is associated with the vortices becoming detached from the tube and convect away from their source. The acoustic particle velocity at which the transition occurs between these regimes has been shown to depend on the geometry of the inner edge of the tune opening. The existence of these two regimes and the associated differences in the energy losses provide a physical understanding of existing termination impedance measurements.

\section{ACKNOWLEDGMENTS}

The author J.M.B. would like to acknowledge the financial support of l'université du Maine which supported his stay as a visiting professor. The author M.A. would like to acknowledge the financial support from CNRS during his Ph.D.

${ }^{1}$ L. J. Sivian, “Acoustic impedance of small orifices," J. Acoust. Soc. Am. 7, 94-101 (1935).

${ }^{2} \mathrm{U}$. Ingard and S. Labate, "Acoustic circulation effects and the nonlinear impedance of orifices," J. Acoust. Soc. Am. 22, 211-218 (1950).

${ }^{3}$ G. B. Thurston and C. E. Martin, Jr., "Periodic flow through circular orifices," J. Acoust. Soc. Am. 25, 26-31 (1953).

${ }^{4} \mathrm{G}$. Thurston, L. Hargrove, Jr., and B. Cook, "Nonlinear properties of circular orifices,” J. Acoust. Soc. Am. 29, 992-1001 (1957). 
${ }^{5} \mathrm{G}$. Thurston, "Nonlinear acoustic properties of orifices of varied shapes and edge conditions," J. Acoust. Soc. Am. 30, 452-455 (1958).

${ }^{6}$ R. Panton, and A. Goldman, "Correlation of nonlinear orifice impedance," J. Acoust. Soc. Am. 60, 1390-1396 (1976).

${ }^{7}$ O. Rudenko and S. Soluyan, Theoretical Foundations of Nonlinear Acoustics (Consultant Bureau, New York, 1977), pp. 1-274.

${ }^{8}$ M. R. Stinson and E. A. G. Shaw, "Acoustic impedance of small, circular orifices in thin plates," J. Acoust. Soc. Am. 77, 2039-2042 (1985).

${ }^{9}$ J. P. Dalmont, C. Nederveen, V. Dubos, S. Ollivier, V. Meserette, and E. te Sligte, "Experimental determination of the equivalent circuit of a side hole: Linear and non linear behaviour," Acta. Acust. Acust. 88, 567-575 (2002).

${ }^{10}$ J. Disselhorst and L. V. Wijngaarden, "Flow in the exit of open pipes during acoustic resonance," J. Fluid Mech. 99, 293-319 (1980).

${ }^{11}$ M. Peters, A. Hirschberg, A. Reijnen, and A. Wijnands, "Damping and reflection coefficient measurements for an open pipe at low Mach and low Helmholtz numbers," J. Fluid Mech. 256, 499-534 (1993).

${ }^{12}$ M. Atig, J. P. Dalmont, and J. Gilbert, "Termination impedance of openended cylindrical tubes at high sound pressure level," C. R. Mcanique 332, 299-304 (2004).

${ }^{13}$ A. Powell, "Theory of vortex sound,” J. Acoust. Soc. Am. 36, 177-195 (1964).

${ }^{14}$ H. Levine and J. Schwinger, "On the radiation of sound from an unflanged circular pipe," Phys. Rev. 73, 383-406 (1948).

${ }^{15}$ Y. Nomura, I. Yamamura, and S. Inawashiro, "On the acoustic radiation from a flanged circular pipe,” J. Phys. Soc. Jpn. 15, 510-517 (1960).

${ }^{16}$ J. P. Dalmont, C. Nederveen, and N. Joly, "Radiation impedance of tubes ended with different flanges: Numerical and experimental investigation," J. Sound Vib. 244, 505-534 (2001).

${ }^{17}$ F. Silva, P. Guillemain, J. Kergomard, B. Mallaroni, and A. N. Norris, "Approximation formulae for the acoustic radiation impedance of a cylindrical pipe," J. Sound Vib. 322, 255-263 (2009).

${ }^{18}$ M. Peters and A. Hirschberg, "Acoustically induced periodic vortex shedding at sharp edged open channel ends: Simple vortex models," J. Sound Vib. 161, 281-299 (1993).

${ }^{19} \mathrm{C}$. Schram, "Aeroacoustics of subsonic jets: Prediction of the sound produced by vortex pairing based on particle image velocimetry," $\mathrm{Ph} . \mathrm{D}$. dissertation, Technische Universiteit Eindhoven, Eindhoven, The Netherlands, p. 107 (2003).

${ }^{20}$ D. Crighton, A. Dowling, J. F. Williams, M. Heckl, and F. Leppington, Modern Methods in Analytical Acoustics (Springer-Verlag, London, 1992), p. 30.

${ }^{21} \mathrm{M}$. Howe, "Contribution to the theory of aerodynamic sound, with applications to excess jet noise and the theory of the flute," J. Fluid Mech. 71, 625-673 (1975).

${ }^{22}$ A. Pierce, Acoustics: An Introduction to its Physical Principles and Applications (McGraw-Hill, New York, 1981), pp. 1-200.

${ }^{23}$ M. Howe, "The dissipation of sound at an edge," J. Sound Vib. 70, 407411 (1980).

${ }^{24} \mathrm{D}$. Rockliff, "Application of particle image velocimetry to the measurement of non-linear effects generated by high-intensity acoustic fields," Ph.D. dissertation, University of Edinburgh, Edinburgh, Scotland, pp. 1-208 (2002).

${ }^{25}$ S. Duffourd, D. Marx, and P. Blanc-Benon, "Détermination de la vitesse acoustique instantanée par PIV dans un stack thermoacoustique (Determination of instantaneous acoustic velocity by PIV in a thermoacoustic stack)," Actes du 6e Congrès d'Acoustique, 535-538 (2002).

${ }^{26}$ D. B. Hann and C. A. Greated, "The measurement of flow velocity and acoustic particle velocity using particle-image velocimetry," Meas. Sci. Technol. 8, 1517-1522 (1997).

${ }^{27}$ D. B. Hann and C. A. Greated, "Measurement of the acoustic particle velocity using particle image velocimetry," Acustica 83, 354-358 (1997).

${ }^{28}$ J. P. Sharp, C. A. Greated, C. Gray, and D. M. Campbell, "The measurement of acoustic streaming using particle image velocimetry," Acustica 68, 168-172 (1989)

${ }^{29}$ D. Skulina, D. Campbell, and C. Greated, "Measurement of the termination impedance of a tube using particle image velocimetry," in Proceedings of the Stockholm Music Acoustics Conference 2003 (2003) Stockhom, Sweden, pp. 747-751.

${ }^{30} \mathrm{~J}$. Westerweel, "Digital particle image velocimetry - Theory and applications," Ph.D. dissertation, Delft University Press, Netherlands, pp. 1-237, 1993.

${ }^{31}$ M. Raffel, C. Willert, and J. Kompenhans, Particle Image Velocimetry: A Practical Guide (Springer-Verlag, Berlin, 1998), pp. 1-253.

${ }^{32}$ R. J. Adrian, "Particle-imaging techniques for experimental fluid mechanics," Annu. Rev. Fluid Mech. 23, 261-304 (1991).

${ }^{33}$ I. Grant, "Particle image velocimetry: A review," Proc. Inst. Mech. Eng. C: J. Mech. Eng. Sci. 211, 55-76 (1997).
${ }^{34} \mathrm{~J}$. Tartarin, “Contribution à l'acoustique non-linéaire des tuyaux ouverts (Non-linear acoustics at open tubes)," Ph.D. dissertation, Université de Poitiers, Poitiers, France, pp. 1-176, 1973.

${ }^{35}$ J. Peube, "Etude expérimentale du champ des vitesses à l'embouchure d'un tuyau sonore excité à de fortes amplitudes (Experimentation study of the velocity fields at the open end of a tube excited by high amplitude sound)," J. Phys. (Paris) 40, 346-352 (1979).

${ }^{36}$ D. Skulina, "PIV measurements of sound fields," Ph.D. dissertation, University of Edinburgh, UK, pp 54-122, 2005.

${ }^{37}$ S. Chen and G. D. Doolen, "Lattice Boltzmann method for fluid flows," Ann. Rev. Fluid Mech. 30, 329-364 (1998).

${ }^{38}$ J. M. Buick, C. A. Greated, and D. M. Campbell, "Lattice BGK simulation of sound waves," Europhys. Lett. 43, 235-240 (1998).

${ }^{39}$ J. M. Buick, C. L. Buckley, C. A. Greated, and J. Gilbert, "Lattice Boltzmann BGK simulation of nonlinear sound waves: The development of a shock front," J. Phys. A 33, 3917-3928 (2000).

${ }^{40} \mathrm{~J}$. M. Buick and J. A. Cosgrove, "Investigation of a lattice Boltzmann model with a variable speed of sound,” J. Phys. A 39, 13807-13815 (2006).

${ }^{41}$ J. M. Buick, J. A. Cosgrove, and R. Higham, "Acoustic lattice Boltzmann model for immiscible binary fluids with a species-dependant impedance," Phys. Rev. E 76, 036713 (2007).

${ }^{42}$ D. Haydock and J. M. Yeomans, "Lattice Boltzmann simulations of acoustic streaming," J. Phys. A 34, 5201-5213 (2001).

${ }^{43}$ D. Haydock and J. M. Yeomans, "Lattice Boltzmann simulations of attenuation-driven acoustic streaming," J. Phys. A 36, 5683-5694 (2003).

${ }^{44} \mathrm{~A}$. Wilde, "Calculation of sound generation and radiation from instationary flows," Comput. Fluids 35, 986-993 (2006).

${ }^{45}$ D. R. Rector, M. S. Greenwood, S. Ahmed, S. R. Doctor, G. J. Posakony, and V. S. Stenkamp, "Simulation of ultrasonic-driven gas separations," J. Acoust. Soc. Am. 121, 3446-3452 (2007).

${ }^{46}$ A. R. da Silva and G. P. Scavone, "Lattice Boltzmann simulations of the acoustic radiation from waveguides," J. Phys. A: Math. Theor. 40, 397408 (2007).

${ }^{47}$ S. Succi, The Lattice Boltzmann Equation for Fluid Dynamics and Beyond (Oxford University Press, USA, 2001), pp. 1-308.

${ }^{48}$ D. Wolf-Gladrow, "Lattice-gas cellular automata and lattice Boltzmann models: An introduction," in Lecture Notes in Mathematics, Vol. 1725 (Springer-Verlag, Heidelberg, 2001), pp.1-308.

${ }^{49}$ Y. H. Qian, D. D'Humières, and P. Lallemand, "Lattice BGK models for Navier-Stokes equation,” Europhys. Lett. 17, 479-484 (1992).

${ }^{50}$ P. L. Bhatnagar, E. P. Gross, and M. Krook, "A model for collision processes in gases. I. Small amplitude processes in charged and neutral onecomponent systems," Phys. Rev. 94, 511-525 (1954).

${ }^{51}$ S. Chen, H. Chen, D. Martnez, and W. Matthaeus, "Lattice Boltzmann model for simulation of magnetohydrodynamics," Phys. Rev. Lett. 67, 3776-3779 (1991).

${ }^{52}$ S. Chen, Z. Wang, X. Shan, and G. D. Doolen, "Lattice Boltzmann computational fluid dynamics in three dimensions," J. Stat. Phys. 68, 379-400 (1992).

${ }^{53}$ U. Frisch, D. d' Humières, B. Hasslacher, P. Lallemand, Y. Pomeau, and J. P. Rivet, "Lattice gas hydrodynamics in two and three dimensions," Complex Syst. 1, 649-707 (1987).

${ }^{54} \mathrm{M}$. Neal, "A study of the brass instrument lip reed mechanism using artificial lips and lattice Boltzmann flow simulations," Ph.D. dissertation, University of Edinburgh, Edinburgh, Scotland, pp. 1-187, 2002.

${ }^{55}$ J. Boyd, J. Buick, J. A. Cosgrove, and P. Stansell, "Application of the lattice Boltzmann model to simulated stenosis growth in a two-dimensional carotid artery," Phys. Med. Biol. 50, 4783-4796 (2005).

${ }^{56}$ Z. Guo, C. Zheng, and B. Shi, "An extrapolation method for boundary conditions in lattice Boltzmann method," Phys. Fluids. 14, 2007-2010 (2002).

${ }^{57}$ A. Hirschberg, J. Bruggeman, A. Wijnands, and N. Smits, "The "whistler nozzle" and horn as aero-acoustic sound sources in pipe systems," Acustica 68, 157-160 (1989).

${ }^{58}$ P. Nelson, N. Halliwell, and P. Doak, "Fluid dynamics of a flow excited resonance, Part II: Flow acoustic interaction,” J. Sound Vib. 91, 375-402 (1983).

${ }^{59}$ J. M. Buick, J. A. Cosgrove, H. Eizenhfer, R. Higham, and R. Royles, "Characterization of the bubble cluster and velocity field in the focal region of a lithotripter," New J. Phys. 9, 240 (2007).

${ }^{60} \mathrm{~L}$. V. King, "On the acoustic radiation pressure on spheres," Proc. R. Soc. London Ser. A 147, 212-240 (1934).

${ }^{61}$ J. F. Vignola, Y. H. Berthelot, S. Jones, and J. Jarzynski, "Equation of motion of microparticles in suspension in an insonified medium," J. Acoust. Soc. Am. 92, 332-334 (1992).

${ }^{62}$ A. Melling, "Tracer particles and seeding for particle image velocimetry," Meas. Sci. Technol. 8, 1406-1416 (1997). 